\title{
Salivary gland ultrasonography in primary Sjögren's syndrome from diagnosis to clinical stratification: a multicentre study
}

\author{
Xia Zhang ${ }^{1 \dagger}$, Ruiling Feng ${ }^{1 \dagger}$, Jinxia Zhao ${ }^{2}$, Yu Wang ${ }^{3}$, Juan He${ }^{4}$, Li Liư ${ }^{5}$, Yongjing Cheng ${ }^{6}$, Haihong Yao , \\ Sumei Tang ${ }^{1}$, Jiali Chen ${ }^{1}$, Shanshan Zhang ${ }^{7}$, Zhiyi Zhang ${ }^{3}$, Qingwen Wang ${ }^{4}$, Jing He ${ }^{1 *}$ and Zhanguo $\mathrm{Li}^{1,8^{*}}$
}

\begin{abstract}
Background: To determine the diagnostic accuracy of major salivary gland ultrasonography (SGUS) in primary Sjögren's syndrome (pSS) using the novel Outcome Measures in Rheumatology Clinical Trials (OMERACT) scoring system in a large-scale multicentre study.

Methods: SGUS was conducted for 246 pSS patients, 140 control subjects with conditions other than SS and 27 healthy control subjects. The echostructure features from the parotid and submandibular glands on both sides were graded using the novel OMERACT scoring system. Receiver operating characteristic curves were used to describe the diagnostic accuracy of the scoring system for pSS. The associations between the SGUS and disease characteristics were analysed to evaluate the clinical value of SGUS for pSS.

Results: The US scores in the pSS group were significantly higher than those in the non-pSS group $(p<0.001)$. The level of diagnostic accuracy was comparable with the scores of all four glands ( $A U C=0.908$ ) when only the parotid and submandibular glands on either side were scored ( $A \cup C=0.910,0.904$, respectively). The optimal cut-off value for the left (right) parotid gland and the left (right) submandibular gland was 4, with maximal sensitivity (75.6\% and $77.2 \%$, respectively) and specificity ( $91.6 \%$ and $92.2 \%$, respectively). The pSS patients with positive SGUS results presented a longer disease duration, parotid enlargement, dental loss and higher levels of serological markers, such as anti-SSA, anti-SSB, positive RF, IgG and $\gamma$-globulin\%.
\end{abstract}

Conclusions: SGUS with the OMERACT scoring system yields high sensitivity and specificity, demonstrating high diagnostic feasibility for pSS. The SGUS may have implications for deciding disease severity and treatment efficacy.

Keywords: Sjögren's syndrome, Salivary glands, Ultrasonography, Diagnosis

\section{Background}

Primary Sjögren's syndrome (pSS) is a relatively common systemic autoimmune disease characterized by lymphocytic infiltration and exocrine gland destruction.

\footnotetext{
*Correspondence: hejing1105@126.com; li99@bjmu.edu.cn

${ }^{+}$Xia Zhang and Ruiling Feng contributed equally to this work.

${ }^{1}$ Department of Rheumatology and Immunology, Peking University

People's Hospital, Beijing, China

${ }^{8}$ Peking-Tsinghua Center for Life Sciences, Beijing, China

Full list of author information is available at the end of the article
}

Approximately 30 to $40 \%$ of patients also suffer from extraglandular manifestations of internal organs [1]. Higher risk of developing oncohemalogical disorders, such as non-Hodgkin lymphoma represents pSS pathognomonic hallmarks and become the major threats to patients with pSS $[2,3]$.

Currently, there have existed various imaging techniques in assessing the involvement of salivary glands in individuals with Sjögren's syndrome (SS). However, considering that these techniques are mostly invasive and original author(s) and the source, provide a link to the Creative Commons licence, and indicate if changes were made. The images or other third party material in this article are included in the article's Creative Commons licence, unless indicated otherwise in a credit line to the material. If material is not included in the article's Creative Commons licence and your intended use is not permitted by statutory regulation or exceeds the permitted use, you will need to obtain permission directly from the copyright holder. To view a copy of this licence, visit http://creativecommons.org/licenses/by/4.0/. The Creative Commons Public Domain Dedication waiver (http://creativeco mmons.org/publicdomain/zero/1.0/) applies to the data made available in this article, unless otherwise stated in a credit line to the data. 
costly and can lead to irradiation, they are still not widely used in the assessment of patient outcomes in daily clinical practice. An increasing body of evidence including our previous study suggests that major salivary gland ultrasonography (SGUS) performs well in diagnosing SS, but the pathological features are defined differently across studies which apply distinct scoring systems [4-9]. Recently, to standardize SGUS, the Outcome Measures in Rheumatology Clinical Trials (OMERACT) working group has reached an international expert consensus on SGUS elementary lesion definitions and a novel fourgrade semiquantitative scoring system (grade $0-3$ ) for pSS [10]. The inter-rater and intra-rater reliability rates of the system were good and excellent, respectively $[10,11]$. However, the diagnostic accuracy and feasibility for pSS and non-pSS sicca patients remain to be tested in clinical practice in large cohort studies.

Furthermore, some studies have shown that SGUS is associated with salivary gland swelling, cryoglobulinemic vasculitis, the production of rheumatoid factor (RF), immunoglobulin and germinal centre-like structures in salivary gland biopsies $[5,6,12,13]$. Of note, previous studies have also provided evidence of SGUS changes after rituximab treatment and predicted the efficacy of xerostomia treatment for pSS [14-16]. Therefore, SGUS might be not only useful as a diagnostic tool but also suitable as a predictive and follow-up biomarker for disease activity and the risk of lymphoma. However, the benefit of SGUS for monitoring the natural history, predicting the outcomes and detecting lymphoma has yet to be investigated in daily clinical practice and larger cohort studies [17].

We aimed to evaluate the diagnostic value of the novel OMERACT scoring system for pSS in this large-scale multicentre study. The secondary aim was to investigate the associations between SGUS characteristics and systemic manifestations of pSS.

\section{Methods \\ Study population}

This was a multicentre study with consecutive patients clinically suspected of having SS enrolled from 5 hospitals across different regions of China between January 2018 and December 2019 in daily clinical practice. All patients were aged $\geq 18$ years and underwent a diagnostic workup for pSS according to the American-European Consensus Group (AECG) or American College of Rheumatology (ACR) criteria $[18,19]$. The patients with other rheumatic diseases accompanied with potential SS overlap were excluded at the beginning of the study. Then, SGUS was conducted for 246 pSS patients, 140 patients with SS-related manifestations but without SS and 27 healthy subjects. A flow chart of the study design was presented in Fig. 1. The study was approved by the medical ethics committee of the Institute of Peking, all participants provided written consent, and the study was performed according to the guidelines of the Declaration of Helsinki.

\section{Clinical, serological and immunological assessment}

All patients were subjected to diagnostic workups for pSS, which was performed without knowledge of SGUS results. The questionnaire-based evaluation included assessments of the following parameters: ocular symptoms; oral symptoms; ocular signs (Schirmer I test $<5$ $\mathrm{mm}$ in $5 \mathrm{~min}$, ocular staining score $>4$ according to the van Bijsterveld scoring system); salivary gland involvement as determined using parotid sialography and salivary scintigraphy; positive histopathological examination results of biopsy specimens of the minor salivary gland (MSG) (defined as the number of mononuclear infiltrates containing $\geq 50$ lymphocytes $/ 4 \mathrm{~mm}^{2}$ of glandular tissue); serological tests, including those for anti-SSA, antiSSB, antinuclear antibody (ANA), RF, immunoglobulin G (IgG) and $\gamma$-globulin\% and other systemic manifestations such as cutaneous vasculitis, arthritis, leukopaenia, interstitial lung disease, renal tubular acidosis and peripheral/ central nervous involvement.

\section{Salivary gland ultrasonography}

All patients were examined by grey-scale and colour Doppler US by five well-trained examiners (S.S.Z., J.X.Z, Y.W., L.L., Y.J.C.) from different centres who were blinded to the clinical data. The US system was equipped with a high-resolution linear transducer $(9-12 \mathrm{MHz})$. The bilateral parotid and submandibular glands were scanned. The novel four-grade semiquantitative scoring system (grade 0-3) developed for the OMERACT was employed to assess the echostructure of each gland [10]. The novel four-grade OMERACT semiquantitative scoring system (grade 0-3) was employed to assess the echostructure of each gland. The scores are defined as: grade 0 , normal parenchyma; grade 1 , mild inhomogeneity without anechoic or hypoechoic areas and hyperechogenic bands; grade 2, moderate inhomogeneity with focal anechoic or hypoechoic areas; and grade 3 , severe inhomogeneity with diffuse anechoic or hypoechoic areas occupying the entire gland or a fibrous gland [10].

\section{Statistical analysis}

All statistical analyses were performed using Statistical Package of Social Science (SPSS) software version 16.0. For statistical comparisons, the Mann-Whitney $U$ test or chi-square test was used, as appropriate. Cohen's kappa was used to analyse the concordance between the scores of bilateral salivary glands. Spearman's test was used for 
Consecutive patients with clinically suspected SS enrolled from 5 hospitals between January 2018 and December 2019

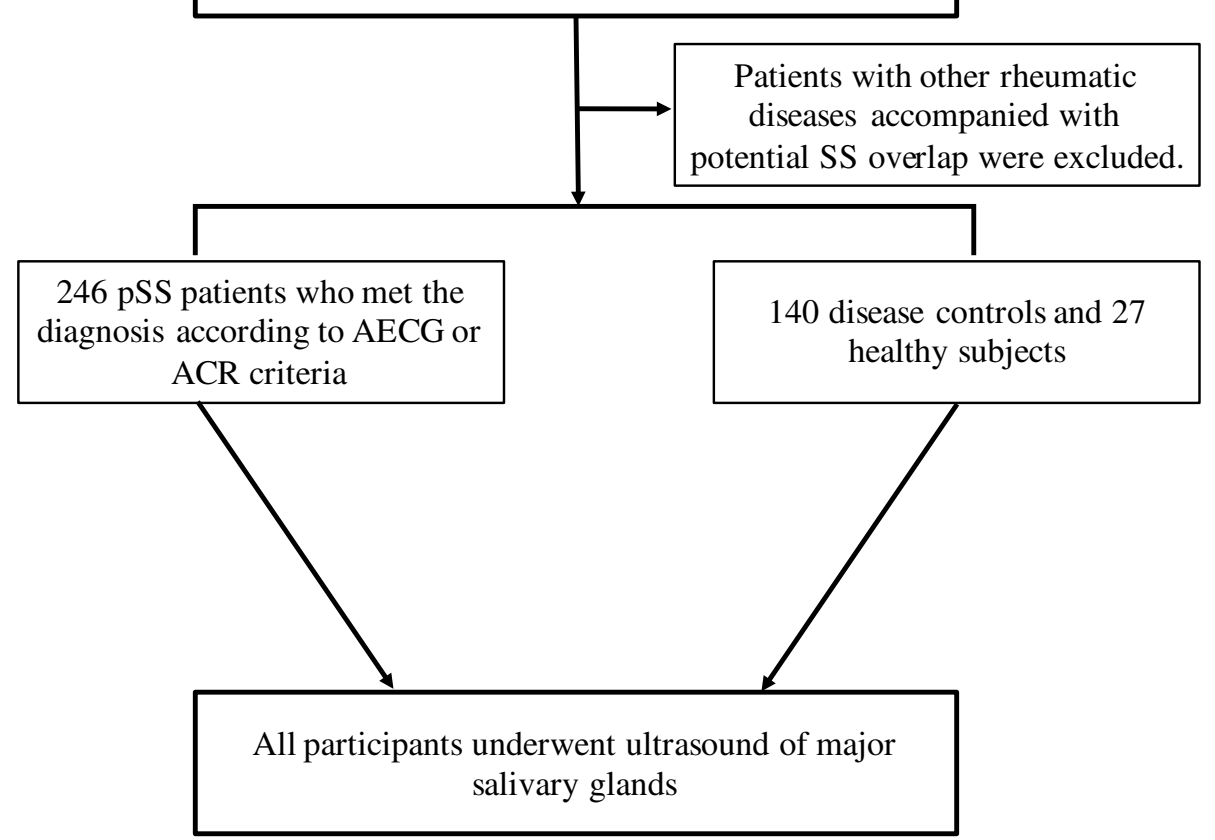

Fig. 1 Flow chart of the study. AECG, American-European Consensus Group; ACR, American College of Rheumatology

correlation analysis. Receiver operating characteristic (ROC) curves were generated to determine the diagnostic performance of the scoring systems for pSS. On the ROC curves, the optimal cut-off point producing the maximal combination of sensitivity and specificity was located nearest the upper left corner of the curve. $P$ values $<0.05$ were considered statistically significant.

\section{Results}

\section{Characteristics of the study population}

A total of 246 patients with pSS who fulfilled the AECG or ACR criteria were included (female/male ratio 239/7; age 53.16 (SD 12.13) years; symptom duration 4 (range $0.1-31)$ years). The control group comprised a total of 167 subjects: 113 non-SS patients with various rheumatic diseases (29 with undifferentiated connective tissue disease, 32 with connective tissue disease, 2 with mixed connective tissue disease, 28 with rheumatoid arthritis, 13 with systemic lupus erythematosus, 1 with sclerosis, 1 with systemic vasculitis, 7 with primary biliary cirrhosis), 27 with idiopathic Sicca syndrome and 27 healthy subjects (female/male ratio 156/11; age 51.21 (S.D. 13.72) years). The two groups were similar regarding age and gender ( $P=0.139$ and 0.068 , respectively). The characteristics of the pSS and non-pSS subjects are shown in Table 1 .

\section{Features of SGUS in pSS}

All patients and control group underwent SGUS scores which displayed no difference between bilateral parotid glands ( $p=0.798)$, followed by bilateral submandibular glands $(p=0.842)$. Further, the kappa values indicated high concordance between the bilateral parotid glands for all participants $(\kappa=0.93, p<0.001)$, so did the bilateral submandibular glands $(\kappa=0.921, p<0.001)$, which indicated that the US scores of the same type of salivary glands were interchangeable (i.e., right vs left parotid gland; right vs left submandibular gland). The US scores of the right parotid and submandibular glands, the scores of the left parotid and submandibular glands and the sum of the scores of all four glands in the pSS group were significantly higher than those in the non-pSS group ( $p<$ 0.001, overall) (Fig. 2).

Correlation analysis suggested a positive correlation between the scores of parotid and submandibular glands on one side and disease duration (Spearman $r=0.208$, $p=0.001)$. Subsequently, to further assess the associations between the SGUS scores and disease duration, the pSS patients were further stratified according to symptom duration [symptom duration: first stage $\leq 5$ years $(n=151)$, second stage $5-10$ years $(n=35)$ and third stage $\geq 10$ years $(n=60)]$. After the analysis based on one side among diverse groups, data showed a trend to 
Table 1 Characteristics of the study population

\begin{tabular}{|c|c|c|c|}
\hline \multirow[t]{2}{*}{ Characteristics } & \multirow{2}{*}{$\begin{array}{l}\text { pSS patients } \\
(n=246)\end{array}$} & \multicolumn{2}{|c|}{ Non-pSS subjects $(n=167)$} \\
\hline & & $\begin{array}{l}\text { Healthy subjects } \\
(n=27)\end{array}$ & $\begin{array}{l}\text { Non-pSS patients } \\
(n=140)\end{array}$ \\
\hline Age, mean (S.D.), years & $53.16(12.13)$ & $51.21(13.72)$ & \\
\hline Female/male & $239 / 7$ & $156 / 11$ & \\
\hline $\begin{array}{l}\text { Symptom duration } \\
\text { Median (range), years }\end{array}$ & $4(0.1-31)^{*}$ & 0 & $2(0.1-30)$ \\
\hline Parotid enlargement & $75(30.5)^{*}$ & 0 & $3(2.1)$ \\
\hline Dental loss & $95(38.6)^{*}$ & 0 & $4(2.9)$ \\
\hline Schirmer $<5 \mathrm{~mm}^{\mathrm{a}}$ & $128 / 129^{*}$ & 0 & $17 / 33$ \\
\hline Anti-SSA ${ }^{a}$ & $176 / 223^{*}$ & 0 & $44 / 130$ \\
\hline Anti-SSB ${ }^{a}$ & $89 / 224^{*}$ & 0 & $15 / 125$ \\
\hline MSG biopsy ${ }^{\mathrm{a}}$ & $37 / 52^{*}$ & 0 & $0 / 1$ \\
\hline
\end{tabular}

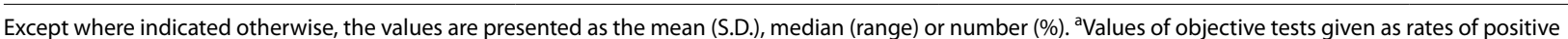
results (positive/total). The $p$ values were determined using the Mann-Whitney test or chi-square test, as appropriate; ${ }^{*} P<0.05:$ statistically significant (regarding the difference between pSS patients and non-pSS subjects). MSG minor salivary gland

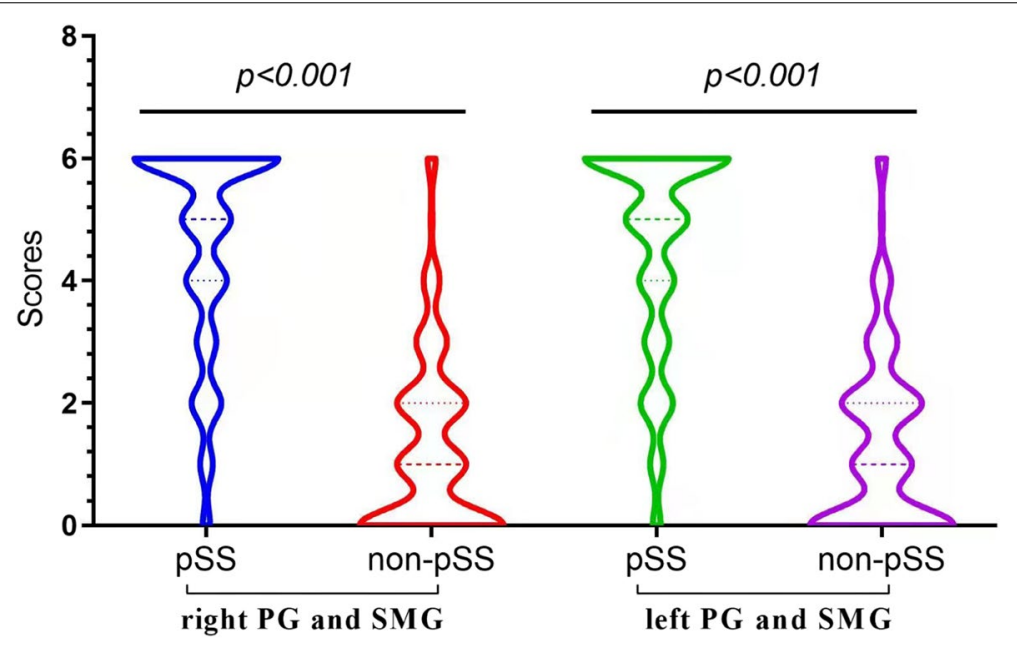

Fig. 2 Features of SGUS scores. Distribution of the US scores for the right parotid and submandibular glands, left parotid and submandibular glands, and all four glands between pSS and non-pSS group. PG, parotid gland; SMG, submandibular gland

have significant differences between the first and second stages and between the first and third stages $(p=0.042$, 0.002 , respectively) but not between the second and third stages $(p=0.345)$ (Fig. 3). Besides, when adjusting SGUS scores to age or gender, there also witnessed no statistical difference among all the subjects in our study.

\section{SGUS diagnostic accuracy for pSS}

The diagnostic accuracy of the US scores of all four glands in diagnosing pSS was excellent (AUC $=0.908$, $95 \%$ CI $0.879,0.938)$. Accuracy was comparable when the scores of only the right parotid and submandibular glands (AUC $=0.910,95 \%$ CI $0.881,0.939$ ) or the scores of only the left parotid and submandibular glands $(\mathrm{AUC}=0.904$,
$95 \%$ CI $0.875,0.934)$ were included ( $p>0.05$, overall) (Table 2). A US cut-off value of 7 provided maximal sensitivity (78\%) and specificity (91.6\%) for the scores of all four glands, while a US cut-off value of 4 showed maximal sensitivity $(77.2 \%$ and $75.6 \%$, respectively) and specificity (92.2\% and 91.6\%, respectively) for the left (right) parotid gland and the left (right) submandibular gland (Table 2), so these values were thought optimal.

\section{SGUS in clinical stratification}

The cut-off value of the SGUS scores of the parotid and submandibular glands on one side allowed for the classification of patients with pSS: the positive SGUS group (SGUS score $\geq 4$ ) and the negative SGUS group (SGUS 


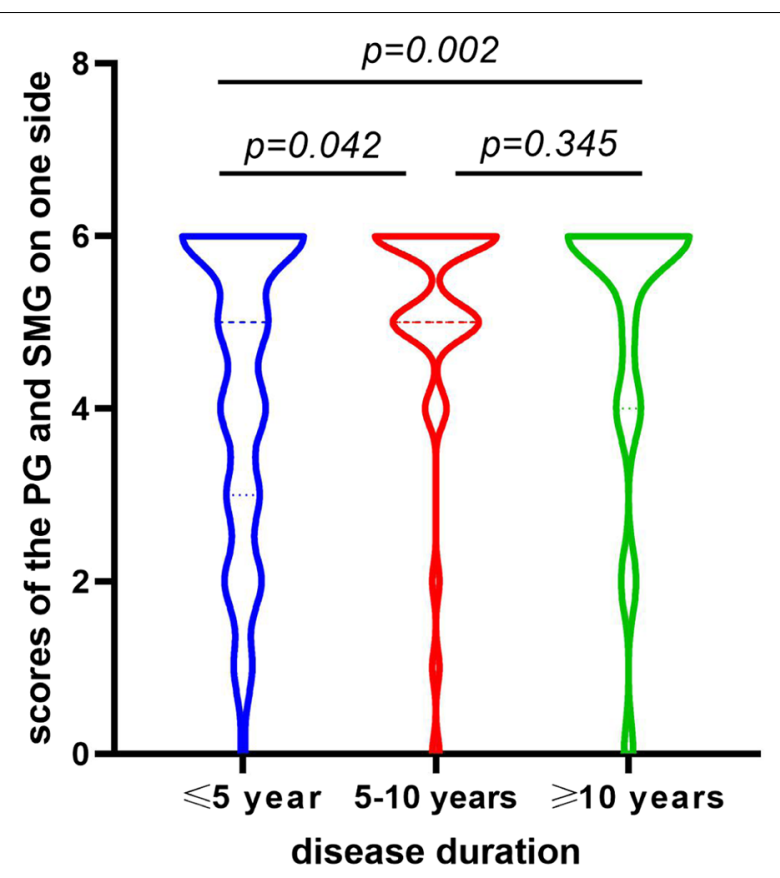

Fig. 3 Association between the scores of the parotid and submandibular glands on one side with disease duration.PG, parotid gland; SMG, submandibular gland

score $<4)$. The results suggested that $190(77.2 \%)$ patients had positive SGUS findings, and 56 (22.8\%) patients had negative SGUS findings. It was observed that the correlation between the positive and negative groups with respect to systemic manifestations including cutaneous vasculitis, interstitial lung disease and other extraglandular manifestations, did not reach significance $(p>$ 0.05 , overall). However, one patient in the SGUS-positive group had cryoglobulinaemia and amyloidosis. Parotid swelling and dental loss were found to occur more frequently in patients with positive scores than in those with negative scores ( $p=0.008,0.001$, respectively) (Table 3 ).
Associations between SGUS and serological parameters In light of the specific value of serological characteristics in diagnosing autoimmune diseases, the pSS patients with positive scores expressed a preference for autoantibodies to SSA and SSB and obviously higher levels of RF, IgG and $\gamma$-globulin\% $(p<0.05$, overall) (Table 3$)$. However, the ANA results along with the level of complement in the positive SGUS group shared the similar information with the negative SGUS group $(p>0.05)$ (Table 3$)$.

Given that the presence of autoantibodies is available for the classification of pSS and daily clinical practice, 239 individuals were evaluated with serum levels of anti-SSA or anti-SSB. Data showed that patients with any of antibodies were significantly involved in the higher SGUS scores $(p<0.05)$ (Fig. $4 \mathrm{~A})$. Additionally, all the samples with positive antibodies were distributed into three groups including only anti-SSA, only anti-SSB antibodies and both positive. Our analysis revealed no significant difference among them ( $p=0.982)$ (Fig. 4B).

In our study, fifty-two patients in the pSS group underwent labial gland biopsies. Among the 52 patients, SGUS score $\geq 4$ of the parotid and submandibular glands on one side and positive biopsy findings were recorded in 38/52 $(73.1 \%)$ and $37 / 52(71.2 \%)$ patients, respectively $(p>$ $0.05)$. The absolute agreement between SGUS scores and labial gland biopsy was $67.3 \%(35 / 52)$.

\section{Discussion}

There is a growing number of evidence suggesting that SGUS has become an integral component in a thorough grasp of typical structural abnormalities of major salivary glands in pSS [4-9]. SGUS has the potential to be used for pSS classification and as a follow-up tool, but for these purposes, additional research, standardized procedures and larger cohort studies are needed [17]. As for the multitude of SGUS abnormalities that occur in individuals with $\mathrm{pSS}$, it is challenging to reach a consensus on the definition and scoring method most appropriate for the most common SGUS abnormalities. By offering an

Table 2 Diagnostic accuracy of the OMERACT scoring system for pSS

\begin{tabular}{|c|c|c|c|c|c|c|}
\hline & Area under the curve $(95 \% \mathrm{Cl})$ & Cut-off value & SEN, \% & SPE, \% & PPV, $\%$ & NPV, $\%$ \\
\hline Right PG & $0.90(0.869-0.930)$ & 2 & 77.6 & 88.6 & 91.4 & 72.9 \\
\hline Right SMG & $0.879(0.845-0.912)$ & 2 & 82.9 & 79.6 & 85.7 & 76 \\
\hline Left PG & $0.893(0.861-0.924)$ & 2 & 76.0 & 89.8 & 91.7 & 71.8 \\
\hline Left SMG & $0.874(0.84-0.908)$ & 2 & 83.3 & 77.8 & 84.7 & 76.0 \\
\hline Right PG + SMG ${ }^{a}$ & $0.910(0.881-0.939)$ & 4 & 77.2 & 92.2 & 93.6 & 73.3 \\
\hline Left PG + SMG ${ }^{b}$ & $0.904(0.875-0.934)$ & 4 & 75.6 & 91.6 & 93 & 71.8 \\
\hline Total four glandsc & $0.908(0.879-0.938)$ & 7 & 78 & 91.6 & 93.2 & 73.9 \\
\hline
\end{tabular}

$P<0.05$ determined by the McNemar test was considered statistically significant. a vs $\mathrm{b}, p=0.508 ; \mathrm{b}$ vs $c, p=0.062$, a vs $c, p=0.625$. $P G$ parotid gland, $S M G$ submandibular gland, SEN sensitivity, SPE specificity, PPV positive predictive value, NPV negative predictive value 
Table 3 Characteristics of pSS patients with positive and negative SGUS results

\begin{tabular}{|c|c|c|c|}
\hline & $\begin{array}{l}\text { Negative } \\
\text { (SGUS < 4) }\end{array}$ & $\begin{array}{l}\text { Positive } \\
\text { (SGUS } \geq 4 \text { ) }\end{array}$ & $p$ \\
\hline$N(\%)$ & $56(22.8)$ & $190(77.2)$ & - \\
\hline Age (S.D.), years & $53.16(12.99)$ & $53.16(11.91)$ & 0.92 \\
\hline Female/male & $54 / 2$ & $185 / 5$ & 0.71 \\
\hline Symptom duration, median (range), years & $2(0.1-27)$ & $5(0.1-31)$ & $<0.001$ \\
\hline Parotid swelling & $9(16.1)$ & $66(34.7)$ & 0.008 \\
\hline Dental loss & $11(19.6)$ & $84(44.2)$ & 0.001 \\
\hline$A N A \geq 1: 320$ & $25(48.1)$ & $105(61.8)$ & 0.08 \\
\hline Anti-SSA positivity & $33(66.0)$ & $143(82.7)$ & 0.011 \\
\hline Anti-SSB positivity & $12(24.0)$ & $77(44.3)$ & 0.01 \\
\hline Positive RF & $29(58)$ & $130(81.8)$ & 0.001 \\
\hline $\operatorname{lgG}, g / l$ & $17.1(6.6)$ & $21.37(8.34)$ & 0.001 \\
\hline Y-globulin,\% & $21.74(6.84)$ & $25.62(6.47)$ & 0.01 \\
\hline C3, median (range), g/l & $1.15(0.88-1.49)$ & $0.96(0.64-1.66)$ & 0.07 \\
\hline C4, median (range), g/l & $0.18(0.12-0.38)$ & $0.17(0.12-0.34)$ & 0.951 \\
\hline \multicolumn{4}{|l|}{ Systemic complications } \\
\hline Cutaneous vasculitis & $6(10.7)$ & $25(13.2)$ & 0.628 \\
\hline Interstitial lung disease & $14(25)$ & $50(26.3)$ & 0.844 \\
\hline Renal involvement & $3(5.4)$ & $20(10.5)$ & 0.243 \\
\hline Nervous system involvement & $3(5.4)$ & $10(5.3)$ & 0.978 \\
\hline Leukopaenia & $14(25)$ & $59(31.1)$ & 0.384 \\
\hline Cryoglobulinaemia & 0 & $1(0.5)^{\mathrm{a}}$ & 0.586 \\
\hline
\end{tabular}

Except where indicated otherwise, the values are presented as the mean (S.D.), median (range) or number (\%). The $p$ values were determined using the Mann-Whitney test or chi-square test, as appropriate. ${ }^{a}$ One patient had cryoglobulinemia and amyloidosis. $P G$ parotid gland, SMG submandibular gland, $A N A$ antinuclear antibodies, $R F$ rheumatoid factor, IgG immunoglobulin G, C3 complement 3, C4 complement 4
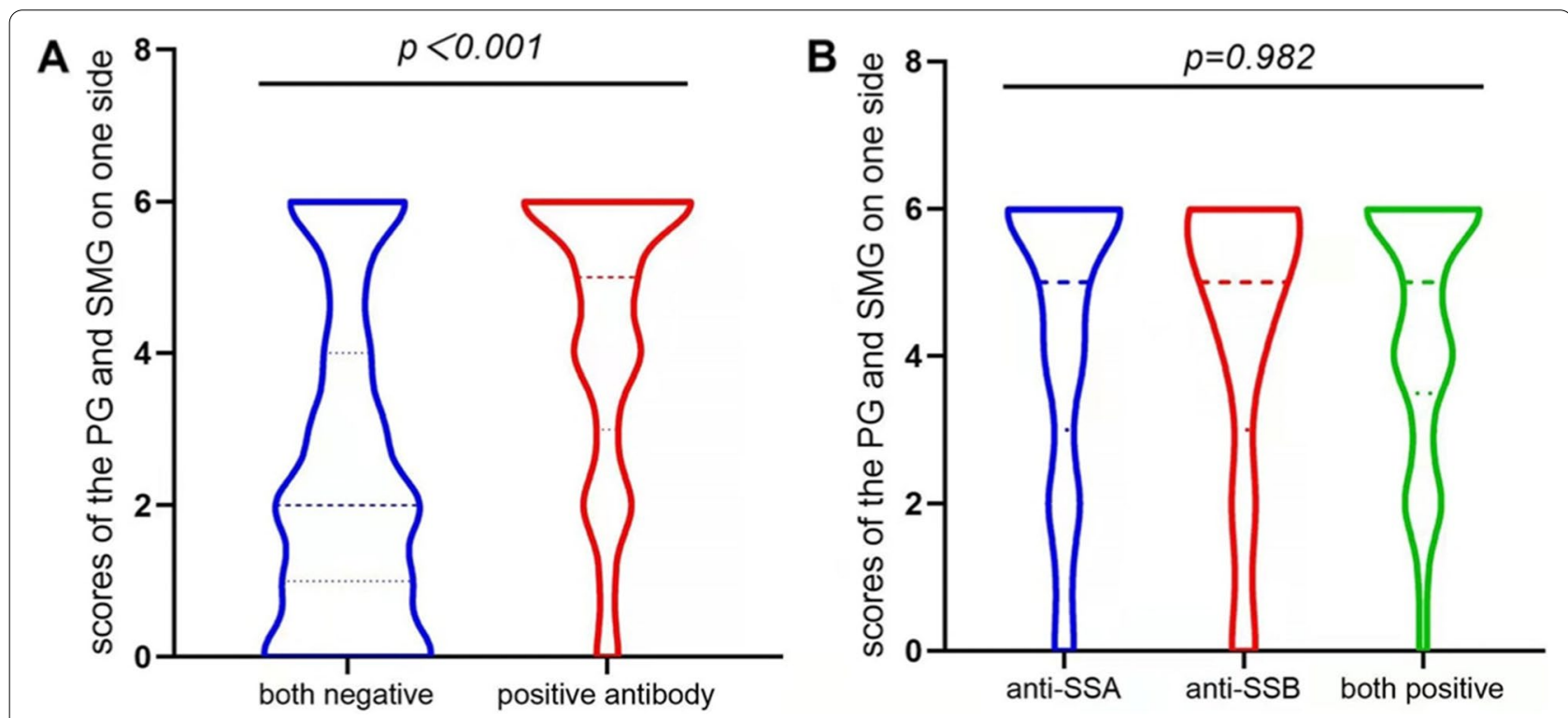

Fig. 4 SGUS scores associated with the existence of any of antibodies. A patients with any positive antibodies compared to both negative. B No significant difference among groups including only anti-SSA, only anti-SSB antibodies and both positive 
updated classification system, a novel four-grade semiquantitative scoring system, the OMERACT group undeniably advanced the diagnosis of pSS [10]. Its intra-rater reliability was excellent and inter-rater reliability was good $[10,11]$. In the next step, the new scoring system needed to be tested in a large number of patients with pSS and non-pSS sicca symptoms.

In this multicentre large-scale study, we confirmed the high diagnostic performance of SGUS to discriminate pSS and symptomatic sicca control patients with the new OMERACT scoring system. The diagnostic accuracy of the US scores of all four glands to diagnose pSS was outstanding and embraced comparable results while only depending on the scores of the parotid and submandibular glands on one side. There was an apparently high correlation between the same type of salivary glands. Our previous study showed that the scores for the submandibular glands on both sides were significantly higher than those for the parotid gland, indicating that there was a weak association between the parotid and submandibular glands [5]. Similar results were reported by Mossel E et al. [20]. An optimal US cut-off value of 7 provided maximal sensitivity (78\%) and specificity (91.6\%) for the scores of all four glands, while an optimal US cut-off value of 4 showed comparable sensitivity $(77.2 \%$ and $75.6 \%$, respectively) and specificity (92.2\% and $91.6 \%$, respectively) for the left (right) parotid gland and the left (right) submandibular gland. Fana V et al. reported that the best ultrasound cut-off value for diagnosing pSS was $\geq 1$ gland with a score $\geq 2$ (sensitivity $=72 \%$, specificity $=91 \%$ ), in which the diagnostic accuracy was similar to our results [21]. Thus, this new scoring system for the left (right) parotid gland and the left (right) submandibular gland showed high diagnostic performance and greatly increased the feasibility of SGUS as a routine procedure in pSS. Given that some patients have unilateral complaints or a high risk of lymphoma, such as parotid enlargement, a global view of all four glands should be taken into consideration, but only one side might be feasible to be scored if there was no particular disease to differentiate.

In addition to the diagnostic value of SGUS, previous studies have shown a correlation between SGUS characteristics and the clinical and serological features of pSS, indicating that SGUS may hold promise for assessing disease activity and treatment efficacy [5, 6, 22]. However, investigations of its usefulness for monitoring the natural history, predicting the outcomes and detecting lymphoma are extremely challenging to perform. In this large-scale multicentre study, we found that the SGUS scores were related to disease duration, parotid swelling, dental loss and the levels of anti-SSA, anti-SSB, RF, IgG and $\gamma$-globulin\%. Cryoglobulinaemia (a well-known risk factor for progression to B lymphoma) and amyloidosis were present in one patient in the SGUS $\geq 4$ group, and no cases of cryoglobulinamia were found in patients with negative SGUS findings. Thus, SGUS was deemed to be related to the usual risk factors for lymphoma of parotid swelling and B cell hyperactivity, regarding polyclonal or monoclonal hyperglobulinaemia and the production of RF. Consistent with the findings of previous studies, Theander and Mandl showed that SGUS enabled patients with systemic complications, high disease activity and lymphoma development to be identified [6]. In addition, Guillaume et al focused on the association between SGUS characteristics and systemic complications and found that SGUS exhibited great benefit in supervising pSS patients with cryoglobulinemic vasculitis or lymphoma [12]. This provides the evidence that SGUS features might be evaluated as a follow-up strategy and may be predictive markers for lymphoma. No cases of lymphoma were found in any of the pSS patients in the present study, probably since the follow-up period was not sufficiently long. Therefore, future longitudinal studies for SGUS should recruit a larger cohort and monitor the risk of lymphoma over time during the natural course of the disease to shed more light on this challenging issue.

Salivary gland abnormalities due to pSS are believed to progress over time, resulting in changes in SGUS features. However, SGUS scores have not been found to be affected by disease duration. Pierre et al. observed that SGUS abnormalities were already present at diagnosis while did not change substantially over the first few years in patients with pSS [23]. Kyung-Ann et al. reported no significant differences between pSS patients with a disease duration $\leq 5$ years and those with a disease duration $>5$ years with respect to the SGUS scores [22]. These findings suggest that pSS may remain asymptomatic for many years, exert a considerable burden on interpreting the time of disease onset. In the present large-scale study, statistically significant differences were observed between disease durations $\leq 5$ years and $5-10$ years and between $\leq 5$ and $\geq 10$ years, but not between $5-10$ and $\geq 10$ years. SGUS abnormalities of hypoechoic areas seem detectable at the early stages of the disease, while diffuse inhomogeneity with anechoic/hypoechoic areas or fibrosis is found in end-stage pSS. According to the novel OMERACT scoring system, SGUS abnormalities with diffuse anechoic/hypoechoic areas or salivary gland fibrosis were defined as grade 3 abnormalities [10]. Therefore, the new scoring system enjoys an effective position on managing well-established diseases at the time of diagnosis whereas restricted in discriminating pSS cases in the middle and late stages.

Importantly, our study did have certain limitations that firstly, some of the SGUS images were scored retrospectively because the novel OMERACT scoring system was 
published online in April 2019. Moreover, we did not follow up patients to monitor the development of lymphoma after the ultrasound examination. Future longitudinal large-scale studies for SGUS are warranted to determine the risk of lymphoma throughout the natural course of the disease.

\section{Conclusions}

In conclusion, this large-scale multicentre study showed that SGUS using the novel OMERACT scoring system demonstrated high sensitivity and specificity for the diagnosis of pSS with good feasibility. This method is also of great value for monitoring disease characteristics. Therefore, SGUS may serve as a widely-adopted approach in the diagnosis, global follow-up and management of pSS.

\begin{abstract}
Abbreviations
pSS: Primary Sjögren's syndrome; SGUS: Salivary gland ultrasonography; OMERACT: Outcome Measures in Rheumatology Clinical Trials; RF: Rheumatoid factor; AECG: American-European Consensus Group; ACR: American College of Rheumatology; MSG: Minor salivary gland; ANA: Antinuclear antibody; IgG Immunoglobulin G; SPSS: Statistical Package of Social Science; ROC: Receiver operating characteristic; PG: Parotid gland; SMG: Submandibular gland; SEN: Sensitivity; SPE: Specificity; PPV: Positive predictive value; NPV: Negative predictive value; $\mathrm{C} 3$ : complement 3 ; $\mathrm{C} 4$ : complement 4 .
\end{abstract}

\section{Acknowledgements}

Not applicable.

\section{Authors' contributions}

All authors of the manuscript fulfil the criteria of authorship. All authors read and approved the final manuscript. X.Z. and J.H. conceived the study. H.H.Y., S.M.T., J.H. and J.L.C. helped design the study. SGUS was performed by S.S.Z., J.X.Z, Y.W., L.L., and Y.J.C. The manuscript was drafted by X.Z. and R.L.F and reviewed and revised by J.X.Z., Z.Y.Z., Q.W.W., Y.J.C, J.H and Z.G.L.

\section{Funding}

This work was supported by the National Natural Science Foundation of China (No. 82071813 and 81601417) and Peking University People's Hospital Research and Development Funds (RS2020-01).

\section{Availability of data and materials}

All relevant generated data and material are included in the manuscript.

\section{Declarations}

\section{Ethics approval and consent to participate}

All participants gave written informed consent, and the study was approved by the medical ethics committee of the Institute of Peking.

\section{Consent for publication}

None to declare.

\section{Competing interests}

The authors declare that they have no competing interests.

\footnotetext{
Author details

'Department of Rheumatology and Immunology, Peking University People's Hospital, Beijing, China. ${ }^{2}$ Department of Rheumatology and Immunology, Peking University Third Hospital, Beijing, China. ${ }^{3}$ Department of Rheumatology and Immunology, Harbin Medical University First Hospital, Harbin, China. ${ }^{4}$ Department of Rheumatology and Immunology, Peking University Shenzhen Hospital, Shenzhen, China. ${ }^{5}$ Department of Ultrasound, Peking University Shenzhen Hospital, Shenzhen, China. ${ }^{6}$ Department of Rheumatology
}

and Immunology, Beijing Hospital, Beijing, China. ${ }^{7}$ Department of Ultrasound, Peking University People's Hospital, Beijing, China. ${ }^{8}$ Peking-Tsinghua Center for Life Sciences, Beijing, China.

Received: 28 June 2021 Accepted: 30 November 2021

Published online: 20 December 2021

\section{References}

1. Mariette $\mathrm{X}$, Criswell LA. Primary Sjogren's syndrome. N Engl J Med. 2018;378(10):931-9.

2. Jonsson MV, Theander E, Jonsson R. Predictors for the development of non-Hodgkin lymphoma in primary Sjogren's syndrome. Presse Med. 2012;41(9 Pt 2):e511-6.

3. De Vita S, Gandolfo S. Predicting lymphoma development in patients with Sjogren's syndrome. Expert Rev Clin Immunol. 2019;15(9):929-38.

4. Hocevar A, Ambrozic A, Rozman B, Kveder T, Tomsic M. Ultrasonographic changes of major salivary glands in primary Sjogren's syndrome. Diagnostic value of a novel scoring system. Rheumatology (Oxford). 2005;44(6):768-72.

5. Zhang $X$, Zhang S, He J, Hu F, Liu H, Li J, et al. Ultrasonographic evaluation of major salivary glands in primary Sjogren's syndrome: comparison of two scoring systems. Rheumatology (Oxford). 2015;54(9):1680-7.

6. Theander E, Mandl T. Primary Sjogren's syndrome: diagnostic and prognostic value of salivary gland ultrasonography using a simplified scoring system. Arthritis Care Res. 2014;66(7):1102-7.

7. Mossel E, Delli K, van Nimwegen JF, Stel AJ, Kroese FGM, Spijkervet $\mathrm{FKL}$, et al. Ultrasonography of major salivary glands compared with parotid and labial gland biopsy and classification criteria in patients with clinically suspected primary Sjogren's syndrome. Ann Rheum Dis. 2017;76(11):1883-9.

8. Takagi Y, Sumi M, Nakamura H, Iwamoto N, Horai Y, Kawakami A, et al. Ultrasonography as an additional item in the American College of Rheumatology classification of Sjogren's syndrome. Rheumatology (Oxford). 2014;53(11):1977-83

9. Martel A, Coiffier G, Bleuzen A, Goasquen J, de Bandt M, Deligny C, et al. What is the best salivary gland ultrasonography scoring methods for the diagnosis of primary or secondary Sjogren's syndromes? Joint Bone Spine : revue du rhumatisme. 2019:86(2):211-7.

10. Jousse-Joulin S, D'Agostino MA, Nicolas C, Naredo E, Ohrndorf S, Backhaus M, et al. Video clip assessment of a salivary gland ultrasound scoring system in Sjogren's syndrome using consensual definitions: an OMERACT ultrasound working group reliability exercise. Ann Rheum Dis. 2019;78(7):967-73.

11. Finzel $S$, Jousse-Joulin S, Costantino F, Hánová P, Hocevar A, lagnocco A et al. Patient-based reliability of the outcome measures in rheumatology (OMERACT) ultrasound scoring system for salivary gland assessment in patients with Sjögren's syndrome. Rheumatology. 2021;60(5):2169-76.

12. Coiffier G, Martel A, Albert JD, Lescoat A, Bleuzen A, Perdriger A, et al. Ultrasonographic damages of major salivary glands are associated with cryoglobulinemic vasculitis and lymphoma in primary Sjogren's syndrome: are the ultrasonographic features of the salivary glands new prognostic markers in Sjogren's syndrome? Ann Rheum Dis. 2021;80(7):e111.

13. Jousse-Joulin S, D'Agostino MA, Hocevar A, Naredo E, Terslev L, Ohrndorf $\mathrm{S}$, et al. Could we use salivary gland ultrasonography as a prognostic marker in Sjogren's syndrome? Response to: 'Ultrasonographic damages of major salivary glands are associated with cryoglobulinemic vasculitis and lymphoma in primary Sjogren's syndrome: are the ultrasonographic features of the salivary glands new prognostic markers in Sjogren's syndrome? 'By Coiffier et al. Ann Rheum Dis. 2021;80(7):e112.

14. Fisher BA, Everett CC, Rout J, O'Dwyer JL, Emery P, Pitzalis C, et al. Effect of rituximab on a salivary gland ultrasound score in primary Sjogren's syndrome: results of the TRACTISS randomised double-blind multicentre substudy. Ann Rheum Dis. 2018;77(3):412-6.

15. Jousse-Joulin S, Devauchelle-Pensec V, Cornec D, Marhadour T, Bressollette L, Gestin S, et al. Brief report: ultrasonographic assessment of salivary gland response to rituximab in primary Sjogren's syndrome. Arthritis Rheum. 2015;67(6):1623-8.

16. Takagi Y, Sumi M, Nakamura H, Sato S, Kawakami A, Nakamura T. Salivary gland ultrasonography as a primary imaging tool for predicting efficacy 
of xerostomia treatment in patients with Sjogren's syndrome. Rheumatology (Oxford). 2016;55(2):237-45.

17. Devauchelle-Pensec V, Zabotti A, Carvajal-Alegria G, Filipovic N, JousseJoulin S, De Vita S. Salivary gland ultrasonography in primary Sjogren's syndrome: opportunities and challenges. Rheumatology (Oxford). 2019:Mar 19:kez079. https://doi.org/10.1093/rheumatology/kez079.

18. Vitali C, Bombardieri S, Jonsson R, Moutsopoulos HM, Alexander EL, Carsons SE, et al. Classification criteria for Sjogren's syndrome: a revised version of the European criteria proposed by the American-European consensus group. Ann Rheum Dis. 2002;61 (6):554-8.

19. Shiboski SC, Shiboski CH, Criswell L, Baer A, Challacombe S, Lanfranchi $\mathrm{H}$, et al. American College of Rheumatology classification criteria for Sjogren's syndrome: a data-driven, expert consensus approach in the Sjogren's international collaborative clinical Alliance cohort. Arthritis Care Res. 2012;64(4):475-87.

20. Mossel E, Arends S, van Nimwegen JF, Delli K, Stel AJ, Kroese FGM, et al. Scoring hypoechogenic areas in one parotid and one submandibular gland increases feasibility of ultrasound in primary Sjogren's syndrome. Ann Rheum Dis. 2018;77(4):556-62.

21. Fana V, Dohn UM, Krabbe S, Terslev L. Application of the OMERACT Greyscale ultrasound scoring system for salivary glands in a single-Centre cohort of patients with suspected Sjögren's syndrome. RMD Open. 2021;7(2):2020-001516.

22. Lee KA, Lee SH, Kim HR. Diagnostic and predictive evaluation using salivary gland ultrasonography in primary Sjogren's syndrome. Clin Exp Rheumatol. 2018;36 Suppl 112(3):165-72.

23. Gazeau P, Cornec D, Jousse-Joulin S, Guellec D, Saraux A, DevauchellePensec $V$. Time-course of ultrasound abnormalities of major salivary glands in suspected Sjogren's syndrome. Joint Bone Spine : revue du rhumatisme. 2018;85(2):227-32.

\section{Publisher's Note}

Springer Nature remains neutral with regard to jurisdictional claims in published maps and institutional affiliations.

- fast, convenient online submission

- thorough peer review by experienced researchers in your field

- rapid publication on acceptance

- support for research data, including large and complex data types

- gold Open Access which fosters wider collaboration and increased citations

- maximum visibility for your research: over 100M website views per year

At BMC, research is always in progress.

Learn more biomedcentral.com/submissions 